\title{
Microfluidics-based capture of human neutrophils for expression analysis in blood and bronchoalveolar lavage
}

\author{
Elizabeth A Warner ${ }^{1}$, Kenneth T Kotz ${ }^{2}$, Ricardo F Ungaro ${ }^{1}$, Amer S Abouhamze ${ }^{1}$, M Cecilia Lopez ${ }^{3}$, Alex G Cuenca ${ }^{1}$, \\ Kindra M Kelly-Scumpia', Claudia Moreno ${ }^{1}$, Kerri A O’Malley ${ }^{1}$, Jennifer D Lanz ${ }^{1}$, Henry V Baker ${ }^{3}$, Larry C Martin ${ }^{1}$, \\ Mehmet Toner ${ }^{2}$, Ronald G Tompkins², Philip A Efron ${ }^{1}$ and Lyle L Moldawer ${ }^{1}$
}

Gene expression analysis can be a powerful tool in predicting patient outcomes and identifying patients who may benefit from targeted therapies. However, isolating human blood polymorphonuclear cells (PMNs) for genomic analysis has been challenging. We used a novel microfluidic technique that isolates PMNs by capturing CD66 ${ }^{+}$cells and compared it with dextran-Ficoll gradient isolation. We also used microfluidic isolation techniques for blood and bronchoalveolar lavage (BAL) samples of patients with acute respiratory distress syndrome (ARDS) to evaluate PMN genomic alterations secondary to pulmonary sequestration. PMNs obtained from ex vivo lipopolysaccharide (LPS)-stimulated or -unstimulated whole blood from five healthy volunteers were isolated by either dextran-Ficoll gradient, microfluidics capture, or a combination of the two techniques. Blood and BAL fluid PMNs were also isolated using microfluidics from seven hospitalized patients with ARDS. Gene expression was inferred from extracted RNA using Affymetrix U133 Plus 2.0 GeneChips. All methods of PMN isolation produced similar quantities of high-quality RNA, when adjusted for recovered cell number. Unsupervised analysis and hierarchical clustering indicated that LPS stimulation was the primary factor affecting gene expression patterns among all ex vivo samples. Patterns of gene expression from blood and BAL PMNs differed significantly from each other in the patients with ARDS. Isolation of PMNs by microfluidics can be applied to both blood and BAL specimens from critically ill, hospitalized patients. Unique genomic expression patterns are obtained from the blood and BAL fluid of critically ill patients with ARDS, and these differ significantly from genomic patterns seen after ex vivo LPS stimulation.

Laboratory Investigation (2011) 91, 1787-1795; doi:10.1038/labinvest.2011.94; published online 19 September 2011

KEYWORDS: dextran; Ficoll; genomics; microfluidics; neutrophil isolation

Still a developing technology, genome-wide expression has emerged as a robust and sensitive tool to study genomic variations among different clinical states, tissues, and cellular mechanisms. To date, widespread use of genome-wide analyses has demonstrated both prognostic capabilities, in both solid and disseminated neoplasms, and predictive utilities for populations at risk for various diseases. More recently, we have shown that unique genome-wide patterns of expression from whole blood are strongly associated with sepsis and multiorgan dysfunction syndrome, ${ }^{1,2}$ and these patterns can be used to identify previously unappreciated signaling pathways important to inflammation. ${ }^{3-9}$ Because of the relative ease and minimal morbidity of obtaining blood samples from a human subject, peripheral blood leukocytes and various sub-populations have taken center stage as an optimal tissue source for genomic analysis in the field of inflammation. ${ }^{10-12}$

Pioneering the field of genomics in trauma has been the 'Inflammation and Host Response to Injury' Large-scale collaborative research program seeking to understand and possibly predict the heterogeneous outcomes associated with major trauma and burn injuries. Through this effort, it has now become clear that the sensitivity of genomic analysis is most powerful when highly enriched cell populations are the source of genomic input rather than mixed-cell populations. ${ }^{10,13}$ Whole blood and solid tissues contain heterogeneous cell populations, and therefore meaningful changes in gene expression patterns can be obscured by conflicting

\footnotetext{
${ }^{1}$ Department of Surgery, University of Florida College of Medicine, Gainesville, FL, USA; ${ }^{2}$ MGH BioMEMS Resource Center, Department of Surgery, Massachusetts General Hospital, Boston, MA, USA and ${ }^{3}$ Department of Molecular Genetics and Microbiology, University of Florida College of Medicine, Gainesville, FL, USA Correspondence: Professor LL Moldawer, PhD, Department of Surgery, University of Florida College of Medicine, Room 6116, Shands Hospital, P.O. Box 100019, 1600 SW Archer Road, Gainesville, FL 32610, USA.

E-mail: moldawer@surgery.ufl.edu
}

Received 3 November 2010; revised 29 March 2011; accepted 30 March 2011 
shifts in leukocyte sub-populations present in the same sample. For this reason, most investigations have evolved toward genomic analyses using enriched leukocyte subpopulations.

Although unintended, cellular enrichment has the potential to introduce genomic artifacts as a result of the isolation procedure. Until recently, the predominant method used to isolate leukocyte populations for genomic analysis has utilized either antibody precipitation and/or sequential centrifugation over discontinuous density gradients (for example, percoll, Ficoll, and dextran), separating cell populations based on differential densities. Unfortunately, these methods are laborious and protracted $(>2 \mathrm{~h})$, and subject the sample to considerable ex vivo perturbations resulting in documented phenotypic and functional changes. ${ }^{14-16}$

In this report, we used a microfluidic cassette capable of rapidly isolating polymorphonuclear leukocytes or neutrophils (PMNs) from biological fluids by antibody capture. This cassette contains a series of branched channels $50 \mu \mathrm{m}$ in height that are coated with a monoclonal antibody to human CD66b (a cell-surface marker specific to granulocytes). Biological fluids are passed through the cassette inlet at optimized flow rates and unbound cells are washed away through a single device outlet, leaving only the adherent $\mathrm{CD} 66 \mathrm{~b}^{+}$cell populations. These adherent cells are then lysed with a chaotrope and nucleic acids are extracted en bloc.

PMNs from unstimulated and ex vivo lipopolysaccharide (LPS)-stimulated whole blood obtained from healthy human subjects were processed in parallel by either dextran-Ficoll sedimentation or through the anti-CD66 ${ }^{+}$-coated microfluidics device. Additionally, an aliquot of PMNs isolated by the dextran-Ficoll method was then subjected to further enrichment using the microfluidic cassette. The extracted RNA from the enriched PMNs obtained from the different isolation procedures was then further processed in parallel for genome-wide expression analysis. In addition, PMNs from whole blood and waste bronchoalveolar lavage (BAL) fluid were also isolated from seven hospitalized patients with acute lung injury (ALI) and/or acute respiratory distress syndrome (ARDS). We demonstrate that PMNs from healthy control subjects have similar genome-wide expression patterns despite the different isolation methods. However, the expression of blood and BAL neutrophils from patients with ALI/ARDS is markedly different from the patterns obtained from unstimulated and ex vivo LPS-stimulated blood PMNs from healthy subjects. These results indicate that the microfluidic isolation procedure appears equivalent to the goldstandard method of PMN isolation for genomic analysis, and can identify differences in gene expression secondary to the source of the PMNs and their in vivo or ex vivo stimuli.

\section{MATERIALS AND METHODS Subjects}

Peripheral venous blood was obtained from five healthy volunteers after obtaining signed informed consent. Venous blood and waste BAL fluid (obtained during a diagnostic procedure) were also obtained from seven critically ill patients with ALI/ARDS, after signed informed consent was provided by their legal representatives. All protocols were approved by the University of Florida, Institutional Review Board before their initiation.

\section{Ex vivo Studies}

Whole blood samples from the healthy control subjects were each divided into two aliquots (see Figure 1). The first aliquot, representing the unstimulated arm, was processed immediately for PMN enrichment. In the second aliquot, the stimulated arm, E. coli (0111:B4) lipopolysaccharide (LPS; Sigma Fine Chemicals, St Louis, MO, USA) was added

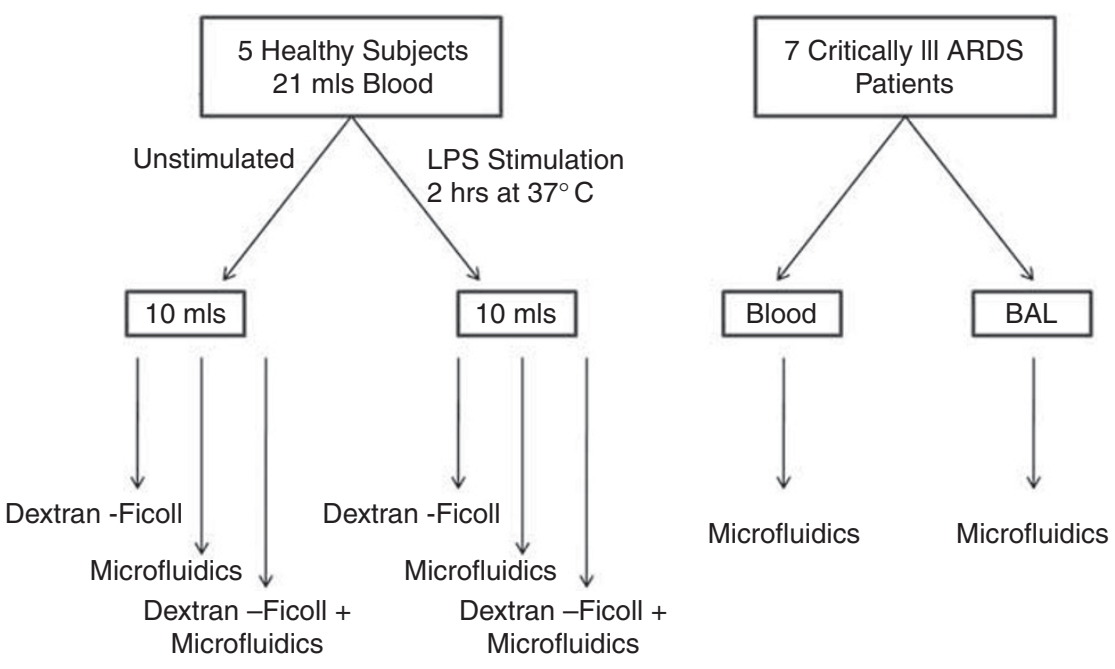

Figure 1 Schematic representation of study designs: ex vivo stimulation of whole blood from healthy subjects, and blood and BAL fluid from hospitalized patients with ARDS. 
at physiological concentrations $(100 \mathrm{ng} / \mathrm{ml})$, and the whole blood was placed in a $5 \% \mathrm{CO}_{2}$ at $37^{\circ} \mathrm{C}$ incubator for $2 \mathrm{~h}$ before PMN isolation. Venous blood $(21 \mathrm{ml})$ from the healthy subjects was collected into Vacutainer tubes containing sodium EDTA (Becton Dickinson, Franklin Lakes, NJ, USA). A 4-ml aliquot of either LPS-stimulated or -unstimulated whole blood was processed by dextran-Ficoll for neutrophil isolation, a $0.35-\mathrm{ml}$ aliquot of whole blood was processed in parallel using the microfluidics cassette, and a 4-ml aliquot of whole blood was processed first by dextran-Ficoll, followed by the microfluidics cassette.

For the ALI/ARDS patients, a single 4-ml blood sample was collected and PMNs were isolated using the microfluidics cassette. In addition, waste BAL fluid (from a diagnostic BAL) was first gravity filtered through a sterile cell strainer (BD Falcon, Bedford, MA, USA) to eliminate particulate debris before PMN isolation using the microfluidics cassette.

\section{Neutrophil Isolation by Dextran-Ficoll Gradients}

Peripheral blood PMNs were isolated by a modified dextranFicoll gradient as previously described by Nauseef et al. ${ }^{17}$ Briefly, the whole blood was diluted with phosphate-buffered saline (PBS) mixed with $2 \%$ fetal calf serum and $100 \mathrm{U} / \mathrm{ml}$ polymixin B. Subsequently, this was layered on top of FicollPaque Plus (Amersham Pharmacia Biotech) and centrifuged at $500 \mathrm{~g}$ for $20 \mathrm{~min}$ at room temperature. The supernatant, interface cells, and Ficoll were then discarded and the remaining neutrophil/erythrocyte suspension was mixed with a $6 \%$ dextran solution and allowed to sediment in a $5 \% \mathrm{CO}_{2}$ incubator at $37^{\circ} \mathrm{C}$ for $30 \mathrm{~min}$. The PMN-enriched clear portion was then transferred to a new tube centrifuged, resuspended in an erythrocyte lysis buffer (EL buffer, Qiagen, Valencia, CA, USA) and kept on ice for $10 \mathrm{~min}$. The sample was then washed with sterile PBS. The PMN pellet was resuspended in RLT buffer (Qiagen) and placed in a QIAshredder spin column (Qiagen) to collect nucleic acids according to the manufacturer's instructions from the lysed PMNs. The RNA was stored at $-70^{\circ} \mathrm{C}$.

\section{PMN Isolation by Microfluidic Cassette}

Microfluidic isolation of PMNs was carried out as previously described. ${ }^{18-20}$ In short, a syringe loaded with $0.35 \mathrm{ml}$ of whole blood or the strained BAL fluid was connected to the inlet and continuously infused into the cassette (Figure 2a) by an automated pump over $5 \mathrm{~min}(30 \mu \mathrm{l} / \mathrm{min})$. During this time, whole blood flowed through the cassette channels while $\mathrm{CD} 6 \mathrm{~b}^{+}$cells (PMNs and eosinophils) were bound to the channel walls through anti-CD66b antibody interactions and other non-CD66 ${ }^{+}$cells (erythrocytes and other leukocytes) remained unbound. After $5 \mathrm{~min}$, a new syringe filled with

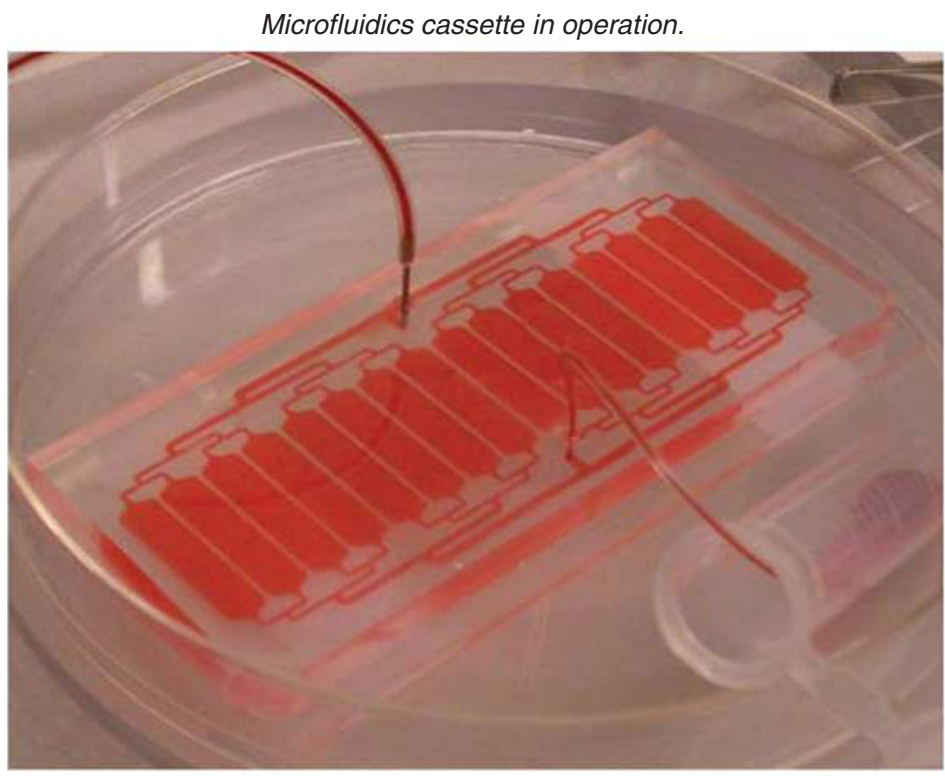

b

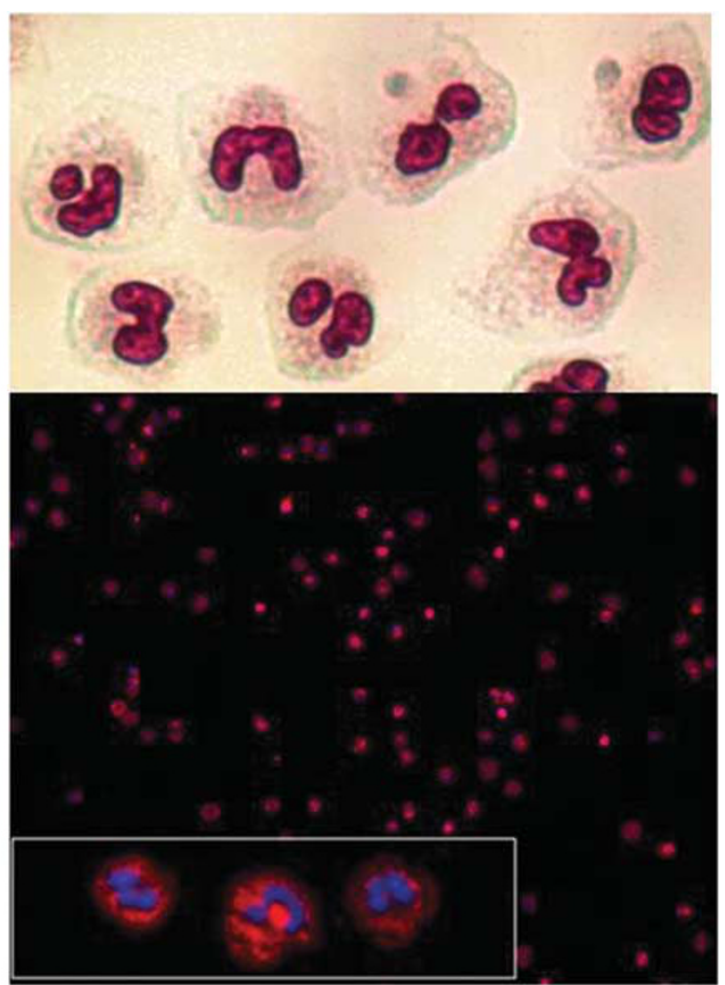

Figure 2 Microfluidics cassette for the enrichment of PMNs from whole blood. (a) Microfluidic cassette with whole-blood filling channels between inlet and outlet ports. (b) Sample purity of captured neutrophils on cassette walls following PBS wash based on Wright-Giemsa and immunofluorescent staining. For fluorescence, cells were labeled with DAPI and PE-labeled CD66 and DAPI staining (magnification $100 \times$ and $10 \times$, respectively). 
PBS, was connected and infused through the cassette for $5 \mathrm{~min}$ at $90 \mu \mathrm{l} / \mathrm{min}$. During this time, unbound cells were washed out of the channels via the outlet port and were discarded while $\mathrm{CD} 66 \mathrm{~b}^{+}$cells $(>95 \%$ PMNs) remained bound to the channel walls. RLT buffer was then infused into the cassette to lyse the adherent cells and then ejected through the outlet channel for the nucleic-acid collection. The cell lysate was then centrifuged over a QIA shredder spin column and the nucleic acids were stored at $-70^{\circ} \mathrm{C}$.

\section{PMN Isolation by Dextran-Ficoll followed by Microfluidics Cassette}

Following isolation of PMNs via dextran-Ficoll sedimentation, the PMN pellet was resuspended in $2 \mathrm{ml}$ sterile PBS, and $350 \mu \mathrm{l}$ of this sample was subsequently subjected to microfluidic processing as described above. Harvested nucleic acids were similarly stored at $-70^{\circ} \mathrm{C}$.

\section{Assessment of Isolated PMN Purity}

The resulting PMN purity from each method was assessed by light microscopy and Wright-Giemsa staining of cytospin preparations from the dextran-Ficoll isolated cells, and cassettes fixed and stained following PMN capture.

\section{RNA Isolation}

Total cellular RNA was isolated using a commercially available kit (RNeasy, Qiagen). RNA concentration and purity were confirmed by capillary electrophoresis (Agilent 2100 Bioanalyser, Agilent).

\section{CDNA Synthesis and Chip Hybridization}

Amplified cDNA was prepared from $\sim 1.5-5 \mathrm{ng}$ of starting PMN RNA using the WT-Ovation Pico RNA Amplification System (NuGen, San Carlos, CA, USA) per the manufacturer's protocol. Subsequently, the cDNA was fragmented and labeled using the FL-Ovation cDNA Biotin Module V2 kit (NuGen) per the manufacturer's protocol. Labeled cDNA was hybridized onto Hg-U133 Plus 2.0 GeneChip oligonucleotide arrays (Affymetrix, Santa Clara, CA, USA), and processed per the manufacturer's protocol with minor modifications.

\section{Microarray Data Analysis}

Chip expression and signal normalization were performed using DNA Chip Analyzer (dChip v1.0.0.1, http:// www.dchip.org) by perfect match algorithms. BRB ArrayTools software was used to analyze data for statistical inference. Differences among groups were evaluated by an adjusted $\mathrm{F}$ test using a false-discovery-adjusted probability (Q) $<0.001$. To assess the ability of individual gene lists to predict treatment assignments, leave-one-out, cross validations were performed (one nearest neighbor, three nearest neighbor, nearest centroid, and diagonal linear discriminate analyses). A Monte Carlo simulation with 1000 permutations of the data set was run and the probability was set at $<0.001$.

\section{RESULTS \\ RNA Quantity/Quality Isolated Using Microfluidics Isolation and Dextran-Ficoll}

RNA quantity and quality were evaluated from blood PMNs isolated by either the microfluidics approach, by dextran-Ficoll density centrifugation, or by a combination of the two techniques. Both techniques generated similar high quantities of nucleic acids for genomic analysis (Table 1). The differences in absolute quantities recovered reflect the differences in starting material ( $4 \mathrm{ml}$ of blood for the dextranFicoll and $0.35 \mathrm{ml}$ for the microfluidics). In fact, the yields when adjusted for starting volumes were remarkably similar, with slightly greater unit yields with the microfluidics. In all cases, the two methods provided more than sufficient RNA for amplification and subsequent hybridization to the GeneChip (Table 1, last column). The purity of the samples was also equivalent with greater than $95 \%$ of the captured cells identified as PMNs by morphology and immunohistochemistry staining (Figure $2 \mathrm{~b}$ ).

\section{Genomic Analysis of Unstimulated and Ex Vivo LPS-Stimulated Whole-Blood PMNs from Healthy Control Subjects}

The primary determinant of gene expression patterns from whole-blood PMNs was exposure to ex vivo LPS stimulation and not the method of PMN isolation. Using an unsupervised approach, 16190 out of the 54645 probe sets had a coefficient of variation of $>0.5$. Hierarchical cluster analysis revealed that the primary separation between the samples was based on whether they were from LPS-stimulated or -unstimulated whole blood (Figure 3). In contrast, clustering based on either the individual patient or method of isolation was less dominant. A subsequent supervised analysis of all samples revealed 7123 probe sets differentially expressed between unstimulated and LPS-stimulated samples using a false-discovery-adjusted probability of $P<0.001$. Additionally, all four leave-one-out cross validation prediction models were able to classify the samples with $100 \%$ accuracy

Table 1 RNA yields and quality using the different isolation procedures

\begin{tabular}{lcccc}
\hline & $\begin{array}{c}\text { RNA } \\
\text { concentration } \\
(\mathrm{pg} / \boldsymbol{\mu})\end{array}$ & $\begin{array}{c}\text { RNA } \\
\text { quantity } \\
(\mathrm{pg})\end{array}$ & RIN & $\begin{array}{c}\text { RNA amp } \\
\text { quantity } \\
(\mathrm{pg})\end{array}$ \\
\hline Method A & $14270^{\mathrm{ab}}$ & $570800^{\mathrm{ab}}$ & 7.78 & $5017^{\mathrm{d}}$ \\
Method B & $1927^{\mathrm{ac}}$ & $69388^{\mathrm{ac}}$ & 8.32 & $4786^{\mathrm{e}}$ \\
Method C & $597^{\mathrm{bc}}$ & $21496^{\mathrm{bc}}$ & 8.5 & $2970^{\mathrm{de}}$ \\
\hline
\end{tabular}

2-tail T-test: $P<0.007^{\mathrm{a}}, P<0.005^{\mathrm{b}}, P<0.005^{\mathrm{c}}, P<0.002^{\mathrm{d}}$, and $P<0.003^{\mathrm{e}}$.

Method A (dextran-Ficoll), method $B$ (microfluidic cassette), method $C$ (dextran-Ficoll followed by microfluidic cassette). The RIN number is obtained from the Agilent Bioanalyzer and is a proprietary measure used to assess the quality of the RNA in terms of degradation products. The values presented here are consistent with a high quality product suitable for microarray analyses. 

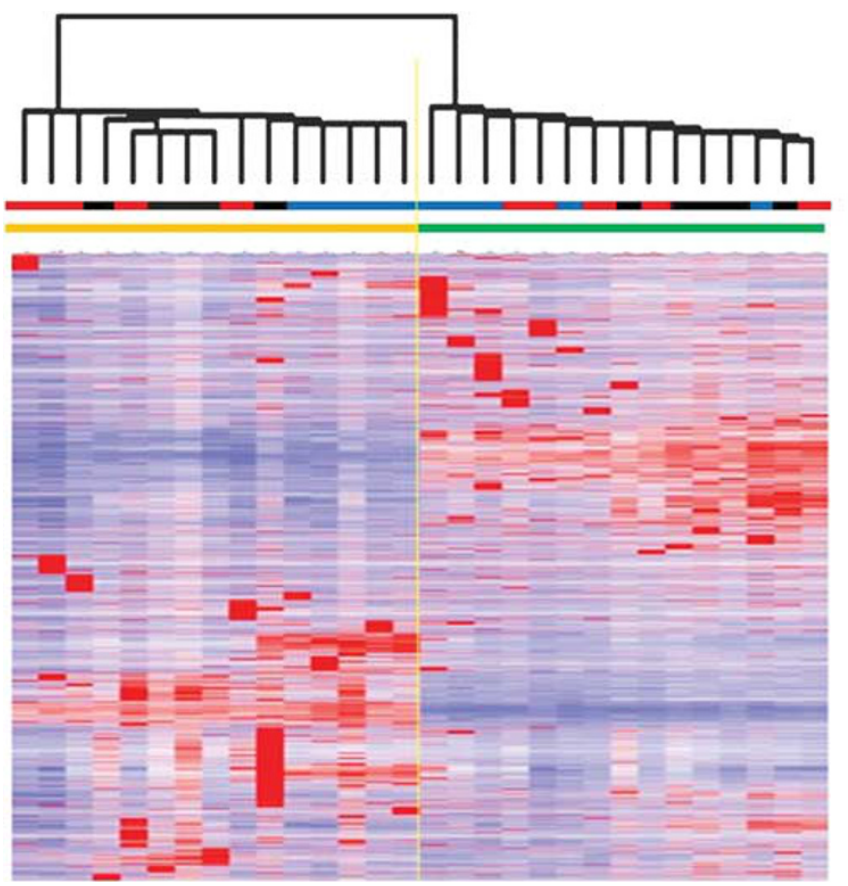

\author{
Source of Blood \\ LPS Stimulated \\ - Unstimulated
}

Method of Isolation

Dextran-Ficoll

Microfluidics Cassette

Dextran-Ficoll +

Microfluidics Cassette

Figure 3 Unsupervised analysis of genome-wide expression. A total of 16190 probe sets achieved a coefficient of variance $>50 \%$ and were used to cluster samples according to similar patterns of gene expression using Pearson's correlation coefficient. The primary separation is distinguished by the presence or absence of ex vivo stimulation by LPS, and not the individual patient or the method of cell isolation.

a
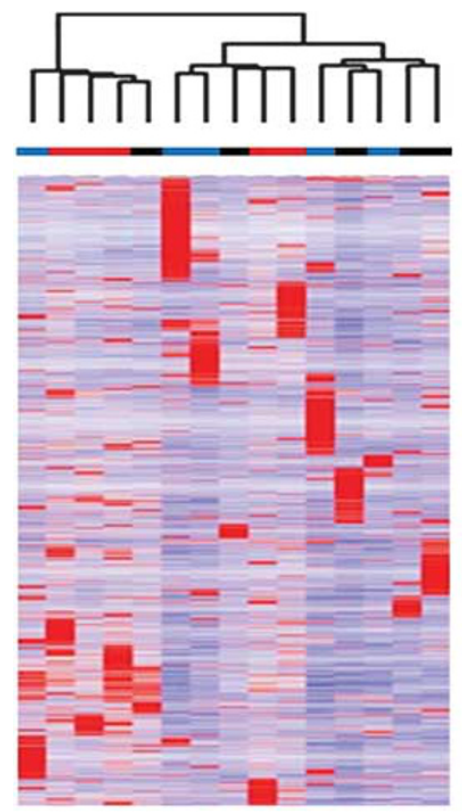

b LPS Stimulated Whole Blood
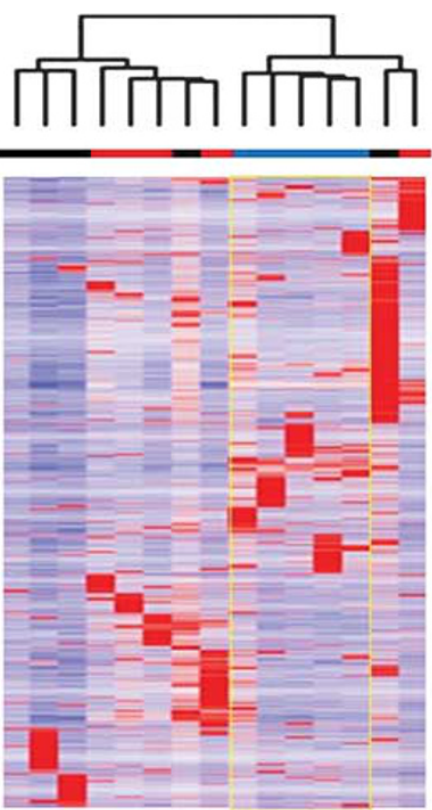

Method of Isolation

Dextran-Ficoll

Microfluidics Cassette

Dextran-Ficoll +

Microfluidics Cassette

Figure 4 Unsupervised analysis of genome-wide expression of unstimulated (a) and LPS-stimulated (b) samples. Unsupervised subanalyses of both the unstimulated samples, where no clustering pattern is readily identified, and LPS-stimulated samples, where microfluidics-based PMN isolation clusters together (see yellow outline).

according to whether they were ex vivo LPS stimulated or unstimulated based on the 7123 probe sets (data not shown).

However, there was some evidence that gene expression patterns could have been affected by the method of isolation, particularly after ex vivo LPS stimulation. As shown in Figure 4, hierarchical clustering within the LPS-stimulated group revealed that samples isolated using dextran-Ficoll (regardless of whether microfluidics was performed) 
clustered more broadly, and the microfluidics isolation alone, clustered more tightly. These findings suggest that the dextran-Ficoll separation introduced some genomic variability in the LPS-stimulated cells. The importance of these findings, however, is unclear because similar clustering based on isolation was not seen in the unstimulated group.

Microfluidic cassette PMN isolation was subsequently used in a clinical setting on samples from seven critically ill patients. Microfluidics isolation of enriched leukocyte populations has rarely been performed on populations obtained from non-blood sources, such as BAL, diagnostic peritoneal lavage, urine or cerebrospinal fluid. Expression patterns in BAL and blood PMNs were compared with expression patterns in whole blood from the healthy controls and the ex vivo LPS-stimulated samples. As shown in Figure 5, a supervised hierarchical cluster analysis revealed that the patterns of gene expression in blood and BAL from ARDS patients were not only distinct from themselves, but were also distinct from PMN gene expression patterns obtained from healthy control subjects, unstimulated, or stimulated ex vivo with LPS. In fact, hierarchical clustering revealed that the gene expression patterns from ARDS patients blood and BAL PMNs were more similar to each other than they were to blood PMNs from healthy control subjects, regardless of whether they were stimulated ex vivo or unstimulated. More importantly, leave-one-out cross validation procedures revealed that the source of the PMNs could be identified with $100 \%$ assurance $(P<0.001)$.

Surprisingly, the differences in the pattern of gene expression between blood and BAL PMNs were not similar to the changes seen with ex vivo LPS stimulation of blood PMNs. As shown in Table 2, selected BAL PMN gene expression was associated with marked decreases in the expression of PMN granular proteins (myeloperoxidase, neutrophil collagenase, NGAL, and lactoferrin), increased expression of chemokines (CXCL1 (Gro- $\alpha)$, CXCL2 $(\mathrm{MIP}-2 \alpha)$, CXCL3 (MIP-2 $\beta)$, CXCL5 (ENA-78), CXCL16
Performance of classifiers during cross-validation.

\begin{tabular}{|c|c|c|c|c|c|c|c|}
\hline & Array id & Class label & $\begin{array}{c}\text { Mean } \\
\text { Number of } \\
\text { genes in } \\
\text { classifier }\end{array}$ & $\begin{array}{c}\text { Diagonal Linear } \\
\text { Discriminant } \\
\text { Analysis } \\
\text { Correct? }\end{array}$ & $\begin{array}{l}\text { 1-Nearest } \\
\text { Neighbor }\end{array}$ & $\begin{array}{c}\text { 3-Nearest } \\
\text { Neighbors } \\
\text { Correct? }\end{array}$ & $\begin{array}{l}\text { Nearest } \\
\text { Centroid } \\
\text { Correct? }\end{array}$ \\
\hline 1 & ARDS1-BAL & ARDS-BAL & 19101 & YES & YES & YES & YES \\
\hline 2 & ARDS2-BAL & ARDS-BAL & 17338 & YES & YES & YES & YES \\
\hline 3 & ARDS3-BAL & ARDS-BAL & 17390 & YES & YES & YES & YES \\
\hline 4 & ARDS4-BAL & ARDS-BAL & 17578 & YES & YES & YES & YES \\
\hline 5 & ARDS5-BAL & ARDS-BAL & 17961 & YES & YES & YES & YES \\
\hline 6 & ARDS6-BAL & ARDS-BAL & 18076 & YES & YES & YES & YES \\
\hline 7 & ARDS7-BAL & ARDS-BAL & 18095 & YES & YES & YES & YES \\
\hline 8 & ARDS1-Blood & ARDS-Blood & 17761 & YES & YES & YES & YES \\
\hline 9 & ARDS2-Blood & ARDS-Blood & 17344 & YES & YES & YES & YES \\
\hline 10 & ARDS3-Blood & ARDS-Blood & 17249 & YES & YES & YES & YES \\
\hline 11 & ARDS4-Blood & ARDS-Blood & 17583 & YES & YES & YES & YES \\
\hline 12 & ARDS5-Blood & ARDS-Blood & 17092 & YES & YES & YES & YES \\
\hline 13 & ARDS6-Blood & ARDS-Blood & 17136 & YES & YES & YES & YES \\
\hline 14 & ARDS7-Blood & ARDS-Blood & 17594 & YES & YES & YES & YES \\
\hline 15 & Health1-Stim & Health-Stim & 17826 & YES & YES & YES & YES \\
\hline 16 & Health2-Stim & Health-Stim & 16773 & YES & YES & YES & YES \\
\hline 17 & Health3-Stim & Health-Stim & 16590 & YES & YES & YES & YES \\
\hline 18 & Health4-Stim & Health-Stim & 16923 & YES & YES & YES & YES \\
\hline 19 & Health5-Stim & Health-Stim & 16772 & YES & YES & YES & YES \\
\hline 20 & Health1-Unstim & Health-Unstim & 17695 & YES & YES & YES & YES \\
\hline 21 & Health2-Unstim & Health-Unstim & 16876 & YES & YES & YES & YES \\
\hline 22 & Health3-Unstim & Health-Unstim & 17482 & YES & YES & YES & YES \\
\hline 23 & Health4-Unstim & Health-Unstim & 17406 & YES & YES & YES & YES \\
\hline 24 & Health5-Unstim & Health-Unstim & 17169 & YES & YES & YES & YES \\
\hline $\begin{array}{l}\text { percent } \\
\text { ourrect } \\
\text { ification: }\end{array}$ & & & & 100 & 100 & 100 & 100 \\
\hline
\end{tabular}
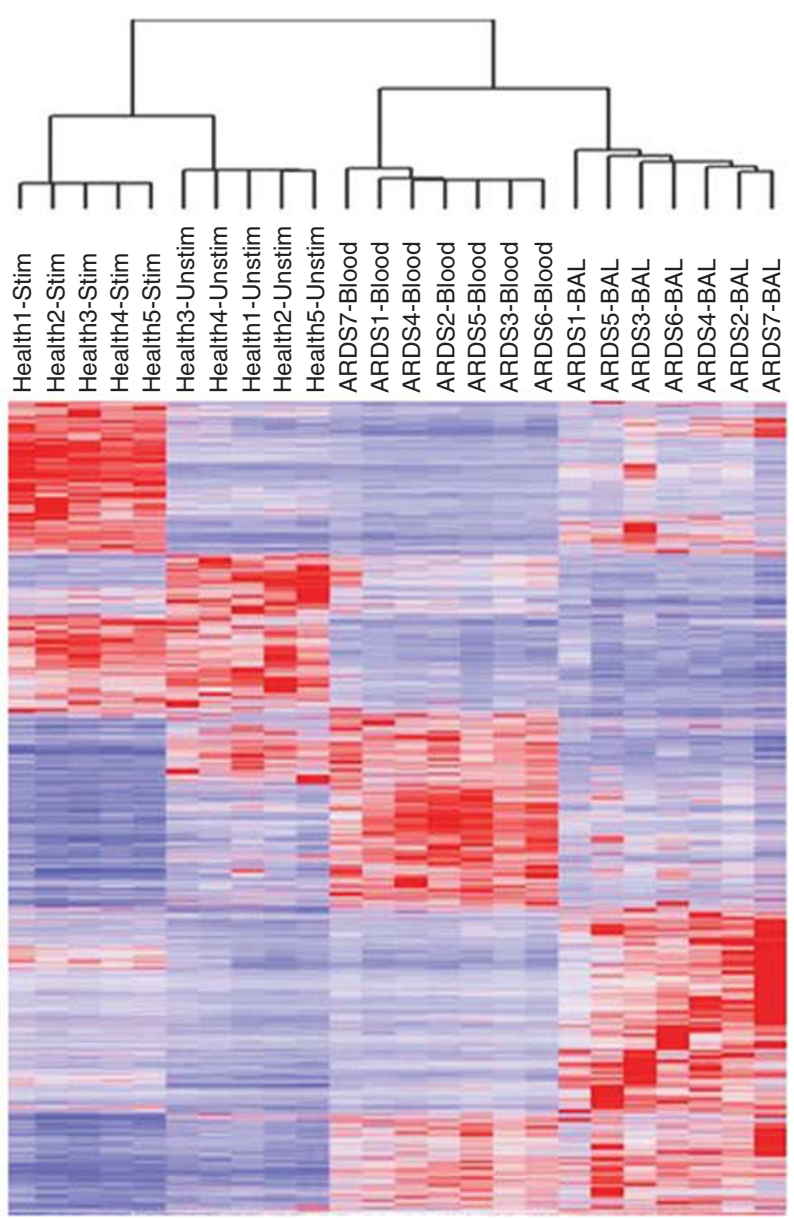

Figure 5 A supervised analysis of gene expression from blood and BAL PMNs isolated from ARDS patients compared with unstimulated and LPS-stimulated whole-blood from healthy control subjects. Differences in gene expression among the four groups were analyzed by F test using a false-discovery-adjusted (Q) probability of $<0.001$. The gene list was then used to determine whether it could accurately predict the group assignment using four different models and leave-one-out cross validation. Each of the four models predicted the classification with $100 \%$ accuracy. $P<0.0001$ using a Monte Carlo simulation with 1000 permutations of the data set. 
Table 2 Selected gene expression patterns in neutrophils obtained from critically ill patients or stimulated ex vivo with LPS

\begin{tabular}{|c|c|c|c|c|c|}
\hline Gene & $\begin{array}{l}\text { Fold change } \\
\text { in BAL }\end{array}$ & $\begin{array}{l}\text { Fold change } \\
\text { due to LPS }\end{array}$ & Gene & $\begin{array}{l}\text { Fold change } \\
\text { in BAL }\end{array}$ & $\begin{array}{l}\text { Fold change } \\
\text { due to LPS }\end{array}$ \\
\hline MPO: myeloperoxidase & $\downarrow 32.4$ & Unchanged & $\begin{array}{l}\text { BAG3: BCL2-associated } \\
\text { athanogene } 3\end{array}$ & $\uparrow 15.2$ (anti-apoptotic) & Unchanged \\
\hline LCN2: lipocalin-2 (NGAL) & $\downarrow 7.2$ & Unchanged & BCLAF1: BTF1 & $\downarrow 2.2$ & Unchanged \\
\hline LTF: Lactoferrin & $\downarrow 23.4$ & Unchanged & & & \\
\hline
\end{tabular}

PMN Chemokines

CXCL1: Gro $\alpha$

CXCL2: MIP2 $\alpha$

CXCL3: MIP2 $\beta$

CXCL5: ENA-78

CXCL16: SRPSOX

$\uparrow 4.8$
$\uparrow 31.1$
$\uparrow 117.8$
$\uparrow 36.2$
$\uparrow 2.5$

Unchanged

$\uparrow 4.8$

$\uparrow 13.8$

Unchanged

Unchanged

Selected genes from BAL were all significantly different from blood of critically ill patients, or LPS stimulated versus unstimulated, using a false-discovery-adjusted probability of $P<0.001$.

(SRPSOX)), and decreased expression of pro-apoptotic proteins (BCL10 and BCLAF1; all $P<0.001)$ compared with blood PMNs from the same patient. In contrast, LPS stimulation of whole-blood PMNs did not recapitulate the changes seen above with the exception that the expressions of CXCL2 and CXCL3 were modestly increased. In contrast, LPS stimulation was more associated with increased expression of genes involved in NF- $\mathrm{KB}, \mathrm{MAPK}$, and oxidative phosphorylation.

\section{DISCUSSION}

As the field of genomics rapidly expands, it has become increasingly clear that standardization of data collection and processing is necessary to extract meaningful insights into the human transcriptome, ${ }^{13,21}$ especially when studying diverse patient populations. The challenge has been to develop methodologies that are not only robust, but also feasible within the clinical setting. Thus, techniques must be rapid, easy to perform, require small blood volumes, and produce meaningful data when performed by skilled nursing and technical staff.

Historically, the most traditional technique to isolate PMNs from whole blood has been density centrifugation using Ficoll and dextran. Unfortunately, these techniques are laborious, challenging, and have the potential to stimulate cell populations through physical perturbation and exposure to multiple solutions throughout the process. We sought to compare the resulting genomic output from the dextran-Ficoll technique with a more rapid microfluidics cassette that is based on the principal of antibody capture. The former technique is characterized by large blood volumes, several hours of processing, and exposure of the cells to gradients and non-physiological solutions, whereas the latter uses very small volumes of blood, is rapid, is processed with physiological solutions, and is easy to perform by research nurses and technicians. ${ }^{20}$ The results suggest that the microfluidics technique can be readily used on samples from hospitalized patients and experimental laboratory blood samples. In fact, under conditions of ex vivo whole-blood stimulation, microfluidics PMN isolation may be associated with less inter-sample variation than with the traditional dextran-Ficoll isolation. Coupled with the small volume requirements, ease of use and low variability, microfluidics methodologies are attractive for the isolation of PMNs for subsequent genome-wide analysis.

The natural question is whether the two techniques give equivalent results. The two isolation strategies are based on fundamentally different properties, and therefore, the resulting cell capture could be very different. With that said, the unsupervised analysis of unstimulated and LPS-stimulated whole-blood PMNs from healthy volunteers (Figure 3) revealed that variations in gene expression patterns were dominantly affected by the presence (or absence) of LPS stimulation and not the individual subject or the method of 
isolation. This suggests that patterns of gene expression introduced by either dextran-Ficoll or microfluidics PMN isolation are less dramatic than the changes in gene expression evoked by ex vivo LPS stimulation. By analyzing the unstimulated and stimulated groups separately, the dramatic genomic variation secondary to LPS stimulation is removed, and the method of isolation effects on the overall patterns of gene expression can be examined. The clustering of samples isolated by only microfluidics in the LPS-stimulated group suggests that PMN microfluidics processing produces the least variable mRNA output of all the tested methods. Whether the outcome of a more homogenous genomic response in the microfluidics group is owing to the introduction of minimal isolation artifact (that is, a more clean method for PMN isolation) or the resulting self-selection of a more similar subset of PMNs (likely mediated by the relative abundance of $\mathrm{CD} 66 \mathrm{~b}^{+}$expression) is unclear. The fact that samples isolated by dextran-Ficoll followed by microfluidics capture clustered more closely with samples isolated by only dextran-Ficoll suggests at a minimum, that the biological and analytical artifacts introduced by microfluidics processing are small, and possibly even less than the variability among subjects or due to the dextran-Ficoll. A supervised analysis of only the stimulated samples identified 387 probe sets ( $Q$ test (false-discovery-adjusted probability) at $P<0.001$ ) significantly different between all three methods which is greater than the number we would expect by chance (with Bonferroni's correction) indicating that there is likely some difference in PMN apparent gene expression due to isolation method, but that that difference is small when compared with the difference generated by ex vivo LPS stimulation (7123 probe sets differentially expressed at $P<0.001$ ).

Previously, we have shown that total leukocytes isolated with microfluidics phenotypically appear to be much less activated than leukocytes subjected to a hypertonic erythrocyte lysis step (as required by dextran-Ficoll isolation). ${ }^{18}$ It is still unclear whether PMN antibody binding to cassette walls via the CD66b receptor induces internal signaling that affects genomic expression. However, the time from PMN binding to cell lysis is less than $10 \mathrm{~min}$ and so any genomic changes would have to be rapid, and as our results indicate, are minimal compared with that of a known PMN stimulus (for example, LPS).

Furthermore, the results presented here suggest that the microfluidics cassettes can be used for genomic analysis of PMN populations not only from blood, but also from BAL fluid. Not surprisingly, the patterns of gene expression from BAL PMNs differed significantly from blood, reflecting reduced apoptosis, increased chemokine expression, and reduced expression of granular contents. However, the patterns of gene expression from BAL PMNs were more similar to the pattern from the blood PMNs of the same subjects, than to the patterns from the blood PMNs stimulated ex vivo with LPS.

\section{Practical Considerations}

Of utmost importance when comparing procedural methods involved in scientific inquiry is the validity and accuracy of the results produced. The results presented here suggest that the two techniques, dextran-Ficoll and microfluidics cassettes are essentially equivalent in generating high-quality nucleic acids by enriching populations of PMNs. However, other considerations, such as time, costs, and ease of application are also important. After working with both macroscale (dextranFicoll) and microscale (microfluidics) PMN separation techniques for genomic analysis, it is clear that microfluidic technology offers multiple distinct advantages: less time required for processing, fewer steps for user error, significantly less sample volume required, and more efficient RNA recovery. Additionally, microfluidics isolation may result in the introduction of less artifactual ex vivo stimulation, as the procedure requires significantly fewer sample manipulations (for example, no centrifugation spins for washing or differential density separation, lack of erythrocyte lysis step), less exposure to non-physiological solutions or $g$ forces, and requires significantly less processing time to nucleic acid recovery.

Importantly, the microfluidics approach can be used in the clinical setting and can be applied to both blood and BAL sources of PMNs. The techniques can clearly distinguish genomic differences between blood and BAL PMNs from critically ill patients, and from blood PMNs between healthy and sick individuals.

\section{ACKNOWLEDGEMENTS}

Supported in part by grants R01 GM-40586, R01 GM-81923 and P41 EB-002503, awarded by the National Institutes of Health, USPHS, KMK-S, AGC, and CM were supported by a National Institute of General Medical Sciences training grant in burns and trauma (GM-008721). AGC was supported by F32 NRSA GM-093665-01, awarded by the National Institute of General Medical Sciences. Development of the microfluidics cassette was supported by the Inflammation and Host Response to Injury Large Scale Collaborative Research Program (Glue Grant; U54 GM062119-9), awarded to Dr Ronald G Tompkins, Massachusetts General Hospital by the National Institute of General Medical Sciences. This work represents a secondary product of the Program, and although the completed manuscript has been seen by the leadership of the Program, the conclusions and discussion are ours, and do not necessarily represent the views of either the Program, Massachusetts General Hospital, or the National Institute of General Medical Sciences.

\section{DISCLOSURE/CONFLICT OF INTEREST}

The authors declare no conflict of interest.

1. Warren HS, Elson CM, Hayden DL, et al. A genomic score prognostic of outcome in trauma patients. Mol Med (Cambridge, Mass) 2009;15: 220-227.

2. Cobb JP, Moore EE, Hayden DL, et al. Validation of the riboleukogram to detect ventilator-associated pneumonia after severe injury. Ann Surg 2009;250:531-539.

3. Alizadeh $A A$, Eisen $M B$, Davis RE, et al. Distinct types of diffuse large B-cell lymphoma identified by gene expression profiling. Nature 2000;403:503-511.

4. Chang JC, Wooten EC, Tsimelzon A, et al. Gene expression profiling for the prediction of therapeutic response to docetaxel in patients with breast cancer. Lancet 2003;362:362-369. 
5. Ayers M, Symmans WF, Stec J, et al. Gene expression profiles predict complete pathologic response to neoadjuvant paclitaxel and fluorouracil, doxorubicin, and cyclophosphamide chemotherapy in breast cancer. J Clin Oncol 2004;22:2284-2293.

6. Balleine $\mathrm{RL}$, Webster $\mathrm{LR}$, Davis $\mathrm{S}$, et al. Molecular grading of ductal carcinoma in situ of the breast. Clin Cancer Res 2008;14: 8244-8252.

7. Chung TP, Laramie JM, Meyer DJ, et al. Molecular diagnostics in sepsis: from bedside to bench. J Am Coll Surg 2006;203:585-598.

8. Feezor RJ, Baker HV, Xiao W, et al. Genomic and proteomic determinants of outcome in patients undergoing thoracoabdominal aortic aneurysm repair. J Immunol 2004;172:7103-7109.

9. Ramilo O, Allman W, Chung W, et al. Gene expression patterns in blood leukocytes discriminate patients with acute infections. Blood 2007; 109:2066-2077.

10. Laudanski K, Miller-Graziano C, Xiao W, et al. Cell-specific expression and pathway analyses reveal alterations in trauma-related human T ell and monocyte pathways. Proc Natl Acad Sci USA 2006;103: 15564-15569.

11. Calvano SE, Xiao W, Richards DR, et al. A network-based analysis of systemic inflammation in humans. Nature 2005;437: 1032-1037.

12. Biberthaler P, Bogner V, Baker HV, et al. Genome-wide monocytic mRNA expression in polytrauma patients for identification of clinical outcome. Shock (Augusta, Ga) 2005;24:11-19.
13. Cobb JP, Mindrinos MN, Miller-Graziano $C_{\text {, }}$ et al. Application of genome-wide expression analysis to human health and disease. Proc Natl Acad Sci USA 2005;102:4801-4806.

14. Watson F, Robinson JJ, Edwards SW. Neutrophil function in whole blood and after purification: changes in receptor expression, oxidase activity and responsiveness to cytokines. Biosci Rep 1992;12:123-133.

15. Eggleton P, Gargan R, Fisher D. Rapid method for the isolation of neutrophils in high yield without the use of dextran or density gradient polymers. J Immunol Methods 1989;121:105-113.

16. Aguado MT, Pujol N, Rubiol E, et al. Separation of granulocytes from peripheral blood in a single step using discontinuous density gradients of Ficoll-Urografin. A comparative study with separation by dextran. $\mathrm{J}$ Immunol Methods 1980;32:41-50.

17. Nauseef WM. Methods in Molecular Biology, Vol. 412: Neutrophil Methods and Protocols, Humana Press Inc.: Totowa, NJ, 2007.

18. Sethu $P$, Moldawer LL, Mindrinos MN, et al. Microfluidic isolation of leukocytes from whole blood for phenotype and gene expression analysis. Anal Chem 2006;78:5453-5461.

19. Russom A, Sethu $P$, Irimia $D$, et al. Microfluidic leukocyte isolation for gene expression analysis in critically ill hospitalized patients. Clin Chem 2008;54:891-900.

20. Kotz KT, Xiao W, Miller-Graziano C, et al. Clinical microfluidics for neutrophil genomics and proteomics. Nat Med 2010;16:1042-1047.

21. Steinmetz LM, Davis RW. Maximizing the potential of functional genomics. Nat Rev 2004;5:190-201. 